TITLE:

\title{
Medial approach for laparoscopic total gastrectomy with splenic lymph node dissection.
}

\section{$\operatorname{AUTHOR}(\mathrm{S}):$}

Okabe, Hiroshi; Obama, Kazutaka; Kan, Takatsugu; Tanaka, Eiji; Itami, Atsushi; Sakai, Yoshiharu

\section{CITATION:}

Okabe, Hiroshi ...[et al]. Medial approach for laparoscopic total gastrectomy with splenic lymph node dissection.. Journal of the American College of Surgeons 2010, 211(1): e1-e6

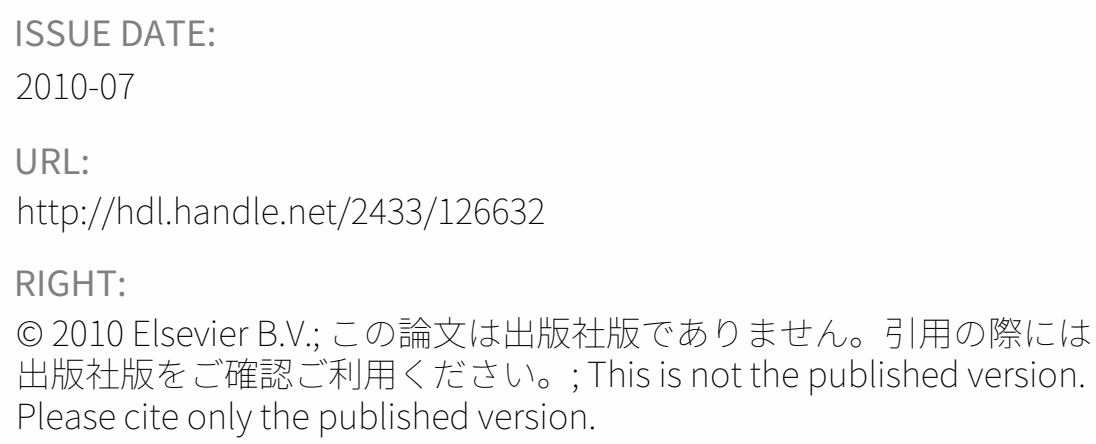

(c) 2010 Elsevier B.V.; この論文は出版社版でありません。引用の際には 出版社版をご確認ご利用ください。; This is not the published version. Please cite only the published version. 
Title:

Medial approach for laparoscopic total gastrectomy with splenic lymph node dissection

Article type: Surgeon at Work

\section{Authors:}

Hiroshi Okabe, MD, PhD, FACS, Kazutaka Obama, MD, PhD, Takatsugu Kan, MD, PhD, Eiji Tanaka MD, PhD, Atsushi Itami, MD, PhD, FACS, Yoshiharu Sakai, MD, PhD, FACS

\section{Affiliation:}

Department of Surgery,

Kyoto University Graduate School of Medicine

54 Kawahara-cho, Shogoin, Sakyo-ku, Kyoto, 606-8507, Japan

\section{Corresponding Author:}

Hiroshi OKABE

Department of Surgery, Kyoto University Graduate School of Medicine588

54 Kawahara-cho, Shogoin, Sakyo-ku, Kyoto, 606-8507, Japan

e-mail:hokabe@kuhp.kyoto-u.ac.jp

phone:+81-75-751-3227, fax:+81-75-751-4390 


\section{Abbreviations:}

LTG, laparoscopic total gastrectomy 


\section{Introduction}

In recent years, laparoscopic distal gastrectomy has become accepted as a surgical option for gastric cancer, which is located in the middle or lower stomach. On the other hand, laparoscopic total gastrectomy (LTG) is less commonly implemented because it requires more complex surgical skills. For the treatment of advanced upper gastric cancer, lymph node dissection along the splenic artery (No. 11) and the splenic hilum (No. 10) is recommended by the Japanese Guidelines. ${ }^{1}$ In open surgery, in order to achieve complete removal of the lymph nodes along the splenic artery, mobilization of the distal pancreas and spleen, as well as the fundus of the stomach, from the retroperitoneum is often performed beforehand. This maneuver allows surgeons to easily dissect lymph nodes in a proximal direction; i.e., from the splenic hilum to the root of the splenic artery. However, within the limited space available during laparoscopic surgery, the same approach for the splenic lymph nodes is not feasible. This paper describes our novel technique of laparoscopic lymph node dissection along the splenic artery by a medial approach, which allows surgeons to easily access the splenic vessels and dissect lymph nodes safely in a distal direction.

\section{Patients and Methods}

\section{Patients}

Between September 2005 and December 2009, we performed total gastrectomy on a total of 126 patients with upper gastric cancer at Kyoto University Hospital. Preoperative diagnoses were made based on gastrointestinal endoscopy, upper gastrointestinal series and abdominal CT scan. Among them, 57 underwent LTG and 69 underwent open total gastrectomy. In 53 of 57 LTG patients, we performed splenic lymph node dissection by the medial approach, while four patients underwent D1 lymph node dissection. The present study included all 53 of these patients who underwent laparoscopic splenic lymph node dissection.

\section{Surgical technique}

The patient is placed in a modified lithotomy position. The first trocar for the videoscope is then inserted via the umbilicus using an open method. Four operating ports and a Nathanson's Liver retractor are inserted as shown in Figure 1. The surgeon stands on the patient's right when mobilizing the fundus of the stomach and dissects the lymph nodes along the splenic artery.

First, the greater omentum is divided to the left until the left gastroepiploic vessels are divided at their roots. Then, splenic hilar lymph node dissection is performed from 
the caudal side. To maintain a good operation field, the patient is placed in a reverse Trendelenburg's position with the left side up. While exposing the branches of splenic vessels, the short gastric vessels are identified and divided at their roots until the uppermost short gastric vessels from the upper polar splenic artery are separated (Figure 2).

Following the dissection of the infrapyloric lymph nodes, the duodenum is transected and the lesser omentum is divided toward the esophago-gastric junction. The phrenoesophageal membrane is then freed from the anterior surface of the esophagus and the gastrophrenic ligament is divided to release the angle of His. The left crus of the diaphragm and the inferior phrenic artery are identified after the dissection (Figure 3a).

Mobilization of the gastric fundus by a medial approach is then initiated. First, we approach the gastrophrenic fold from the right side (Figure $3 b$ ). The fold is cut along the right crus of the diaphragm towards the esophagus. Then, we carefully search for the space between the perigastric fat tissue and the crura of the diaphragm. Advancement of this dissection cephalad and leftward leads us to identify "the white line", which indicates the membranous border between the perigastric tissue and the surface of the retroperitoneum (Figure $3 \mathrm{c}$ ). Further separation of this border allows us to reach the subphrenic free space, and the gastric fundus is completely mobilized. Usually, the fundic branch of the inferior phrenic artery is identified during the dissection. By division of the fundic branch, the esophagus is completely isolated from the crura of the diaphragm. The operative view after the fundic mobilization is shown in Figure $3 \mathrm{~d}$.

Next, we approach the gastropancreatic fold from the caudal side. The perivascular space alongside the left gastric artery is entered bluntly to reach the previously dissected space over the left crus and the retroperitoneum (Figure 4a). The left gastric artery is divided at its root, and the lymph node stations \#8a and \#11p are completely removed (Figure $4 \mathrm{~b}$ ). The detailed technique of lymphadenectomy along the celiac and hepatic arteries is described elsewhere. ${ }^{2}$ After the lymph node station \#9 is dissected and separated from the crus of the diaphragm, the abdominal esophagus is completely exposed by division of the anterior and posterior vagal trunks.

After the esophagus is transected using an endoscopic linear stapler, the assistant retracts the gastric fundus and the gastropancreatic fold caudally to show the membranous border between the retroperitoneum and the posterior aspect of the pancreatic body (Figure 4c). By dissecting this border, the pancreatic body is partially mobilized from the retroperitoneum. This mobilization allows surgeon to approach the distal splenic artery from behind. The retropancreatic fascia is then divided to expose the posterior aspect of the splenic artery and the splenic vein. Finally, the lymph node 
station \#11d along the splenic artery is completely removed in a distal direction and the total gastrectomy is completed (Figure $4 d$ and 5).

The umbilical trocar wound is extended and the resected stomach is removed through it. The proximal margin of the specimen is pathologically examined when necessary. A Roux-en-Y reconstrucion is performed by the previously described technique. ${ }^{3}$ A flat type suction drain is inserted from the upper right port and placed behind the esophago-jejunal anastomosis to the left subphrenic space. The operation is completed by closure of all trocar wounds.

\section{Results}

Patient characteristics

The patients' characteristics are listed in Table 1 . The 53 patients (29 male and 24 female) had a median age of 67 years (range: 30 to 88 years) and a body mass index of 21.4 (range: 16.7 to 29.6 ). Most of the patients were diagnosed preoperatively as stage IA $(n=29)$ or stage IB $(n=17)$. Two patients were diagnosed as stage II and five were diagnosed as stage IIIB.

Surgical outcome

The surgical procedures and outcomes are shown in Table 2. The mean operative time for 53 patients was $359 \pm 76 \mathrm{~min}$, and the estimated blood loss was $187 \pm 186 \mathrm{~g}$. Cholecystectomy was performed concurrently in three patients, who had gallstones. One patient with a T3 tumor located on the greater curvature underwent splenectomy. One patient with a tumor invading the transverse mesocolon underwent transverse colectomy. Combined resection for a duplicated tumor was performed in two patients; lower anterior resection for a rectal cancer, and left nephrectomy for a renal cell carcinoma. One patient with intraoperative esophagojejunal anastomotic trouble required conversion to the open procedure (1.9\%). The mean number of retrieved lymph nodes was $50.8 \pm 19.8$. Regarding the splenic lymph node dissection, complete removal of lymph node station \#11 was performed in 33 patients. In seven of those patients, lymph nodes around the splenic hilum were also dissected. In 13 patients, only lymph nodes along the proximal splenic artery were retrieved. The mean numbers of lymph nodes removed at each splenic lymph node station are indicated in Table 2. All operations were curative except for one patient, in whom peritoneal dissemination was identified during procedure. Postoperative complications were found in 10 patients (18.9\%). Intraabdominal abscess were most frequent and were observed in four patients $(7.5 \%)$. All of them were resolved following treatment with antibiotics. Other complications include pancreatic fistula, pneumonia, wound infection, stasis, angina 
pectoralis and pseudoaneurysm.

The median day that intake of clear liquid was resumed was the third postoperative day and food ingestion was resumed on the following day. The median postoperative hospital stay was 13 days. There was no mortality.

\section{Discussion}

In recent years, as more gastric cancer patients have undergone laparoscopic gastrectomy, surgical techniques for laparoscopic distal gastrectomy have been established. ${ }^{4}$ However, LTG is still technically demanding, because additional procedures are required; such as mobilization of the upper stomach, splenic lymph node dissection, as well as esophagojejunal anastomosis. For esophagojejunal anastomosis, we have reported the safety of the linear stapled technique, which allows us to perform total gastrectomy completely using the laparoscopic approach. ${ }^{3}$ Although some controversies exist, splenic lymph node dissection is recommended for advanced proximal gastric cancer by the Japanese treatment guideline and is routinely performed in Asian countries and specialized centers in the West. ${ }^{1}$ In retrospective comparative studies of open vs. laparoscopic distal gastrectomy, many have reported the achievement of the same extent of lymph node dissection by laparoscopic surgery. ${ }^{5}$ However, difficulties involved with the laparoscopic removal of the second tier lymph nodes, especially of lymph nodes along the splenic artery (station \#11), have also been reported. ${ }^{6}$ The frequencies of lymph node metastasis in stations \#10 and \#11 are higher in upper gastric cancer patients requiring total gastrectomy. Therefore, in order to adopt laparoscopic surgery for upper gastric cancer, we need to establish a safe and efficient technique for laparoscopic splenic lymph node dissection.

In open surgery, complete removal of lymph nodes along the splenic artery can be achieved after mobilization of the distal pancreas and spleen from the retroperitoneum. However, the same approach is hard to apply for laparoscopic surgery because of the limited working space. Thus, we need to develop a novel technique that is suitable for laparoscopic surgery. There have been few studies reporting techniques of laparoscopic splenic lymphadenectomy for gastric cancer. Taping of the splenic artery has been reported as a useful technique by Hur et al. $^{7}$ Others have reported successful distal splenic and splenic hilar lymph node dissection by tilting the operating table left side up, emphasizing the importance of traction by gravity. ${ }^{8}$ When we perform lymphadenectomy along the splenic artery, there is a potential risk of injury of the pancreas or splenic vessels, resulting in uncontrollable intraoperative hemorrhage or postoperative pancreas-related complications. To avoid these injuries, it is critical to identify the total 
running of the splenic artery and its branches to the spleen. Although previous methods might be helpful, it is hard to follow the splenic vessels only from the anterior side, because the splenic artery has many variations of branching and often runs behind the pancreas to some degree.

To solve this problem, we have adopted a medial approach, in which we mobilize the pancreas from the cranial side following fundic mobilization and esophageal transection. This novel approach allows us to identify the total running of the splenic vessels from behind, assuring the safe and precise splenic lymphadenectomy. In our series of patients, we experienced no uncontrolled bleeding from the splenic vessels, which required conversion. After the completion of the lymph node dissection, small splenic ischemia or infarction was seen in some patients. However, no patient developed clinical symptoms, such as high fever or left subcostal pain. The postoperative complication rate was as low, similar to the one report of open gastrectomy with D2 lymph node dissection ${ }^{9}$. These data indicate that the medial approach is useful for secure splenic lymphadenectomy.

Furthermore, complete removal of the splenic lymph nodes, part of which is located behind the pancreas, is easier by our approach than by an approach from the anterior side. The numbers of harvested lymph nodes in LTG were similar to those obtained in open total gastrectomies during the same period (50.8 vs. 53.1), suggesting the oncologic feasibility of our laparoscopic approach to advanced diseases. Although no metastatic lymph node at the splenic hilum was found in this series of patients, splenectomy will be recommended when a positive lymph node is identified during operation. Because the pancreas is already mobilized before the splenic lymph node dissection by our approach, splenectomy will be easily performed. We have successfully accomplished the splenic lymph node dissection in all patients. However, we have not experienced very obese patients in this series. To perform splenic lymph node dissection in highly obese patients, splenectomy might be easier than spleen-preserving lymphadenectomy.

In conclusion, we believe our method could become the standard for LTG with splenic lymph node dissection and facilitate the acceptance of LTG as a surgical option for patients with advanced upper gastric cancer.

\section{References}

1. Japanese Gastric Cancer Association. Guidelines for Diagnosis and Treatment of Carcinoma of the Stomach. April 2004 edition. Tokyo; Kanehara \& CO., LTD.:2004

2. Satoh S, Okabe H, Kondo K, et al. A novel laparoscopic approach for safe and simplified 
suprapancreatic lymph node dissection of gastric cancer. Surg Endosc. 2009;23:436-437.

3. Okabe H, Obama K, Tanaka E, et al. Intracorporeal esophagojejunal anastomosis after laparoscopic total gastrectomy for patients with gastric cancer. Surg Endosc. 2009;23:2167-2171.

4. Tanimura S, Higashino M, Fukunaga $\mathrm{Y}$, et al. Laparoscopic gastrectomy for gastric cancer: experience with more than 600 cases. Surg Endosc. 2008; 22(5):1161-1164.

5. Noshiro H, Nagai E, Shimizu S, Uchiyama A, Tanaka M. Laparoscopically assisted distal gastrectomy with standard radical lymph node dissection for gastric cancer. Surg Endosc. 2005;19:1592-1596.

6. Miura S, Kodera Y, Fujiwara M, et al. Laparoscopy-assisted distal gastrectomy with systemic lymph node dissection: a critical reappraisal from the viewpoint of lymph node retrieval. J Am Coll Surg. 2004;198:933-938.

7. Hur H, Jeon H, Kim W. Laparoscopic pancreas- and spleen-preserving D2 lymph node dissection in advanced (cT2) upper-third gastric cancer. J Surg Oncol. 2008; 97:169-172.

8. Hyung W, Lim J, Song J, Choi S, Noh S. Laparoscopic spleen-preserving splenic hilar lymph node dissection during total gastrectomy for gastric cancer. J Am Coll Surg. Aug 2008;207:e6-11.

9. Sasako M, Sano T, Yamamoto S, et al. D2 lymphadenectomy alone or with para-aortic nodal dissection for gastric cancer. N Engl J Med. 2008;359:453-462. 
Table 1 Summary of patient characteristics

Number of Patients

Median age, years (range)

Male/Female

Body Mass Index

Preoperative stage

IA

IB

II

IIIA

IIIB

IV
53

$67(30-88)$

$29 / 24$

$21.4(16.7-29.6)$

29

17

2

0

5

0 
Table 2 Surgical procedures and outcome

Blood loss (g)

$187 \pm 186$

Operation time ( $\mathrm{min})$

$359 \pm 76$

Combined resection

Gall bladder 3

Spleen 1

Colon 1

Kidney 1

Rectum 1

Extent of splenic lymph node dissection

$\begin{array}{lr}\# 11 p & 13 \\ \# 11 p+\# 11 d & 33 \\ \# 11 p+\# 11 d+\# 10 & 7\end{array}$

Number of dissected lymph nodes

$\begin{array}{lr}\# 10 & 2.6 \pm 2.8 \\ \# 11 \mathrm{p} & 2.6 \pm 2.0 \\ \# 11 \mathrm{~d} & 1.8 \pm 1.7 \\ \text { Total } & 50.8 \pm 19.8\end{array}$

Postoperative complications

Intraabdominal abscess $\quad 4$

Pancreatic fistula 1

Stasis 1

Pneumonia 1

Angina pectoralis 1

Wound infection 1

$\begin{array}{ll}\text { Pseudoaneurysm } & 1\end{array}$ 


\section{Figure Legends}

Figure 1 Port placement for LTG.

A flexible videoscope is inserted through A $(12 \mathrm{~mm})$. Four operating ports for a surgeon and an assistant are inserted through $B$ to $E$ (B: $5 \mathrm{~mm}$; $C$ to $E: 12 \mathrm{~mm})$. A Nathanson's Liver retractor is inserted through $\mathrm{F}$.

Figure 2 Splenic hilar lymph node dissection

Splenic hilar lymph nodes are removed while the short gastric vessels (SGV) are divided at their roots.

Figure 3 Mobilization of the gastric fundus by the medial approach.

a. Release of the gastrophrenic ligament. b. Approach to the gastrophrenic fold. The cut line is shown by the dotted arrow. c. The two arrows show the membranous border between the perigastric fat and the retroperitoneum. $d$. The operative view after the fundic mobilization.

Figure 4 Lymph node dissection along the splenic artery. 
a. Dissection of the perivascular space along the left gastric artery. b. Removal of the proximal splenic lymph nodes (\#11p) from the proximal splenic vessels. c. Pancreatic mobilization. A white dotted line shows the border between the retroperitoneum and the mobilized pancreas, which is covered by the retropancreatic fusion fascia $\left(^{*}\right)$. The splenic vessels run under the fascia. d. Dissection of the distal splenic lymph nodes (\#11d). After division of the retropancreatic fascia, \#11d lymph nodes are dissected while the splenic vessels are exposed. CHA: common hepatic artery, SPA: splenic artery, SPV: splenic vein.

Figure 5 Operative view after the completion of the splenic lymph node dissection CHA: common hepatic artery, SPA: splenic artery, SPV: splenic vein. 
Figure 1

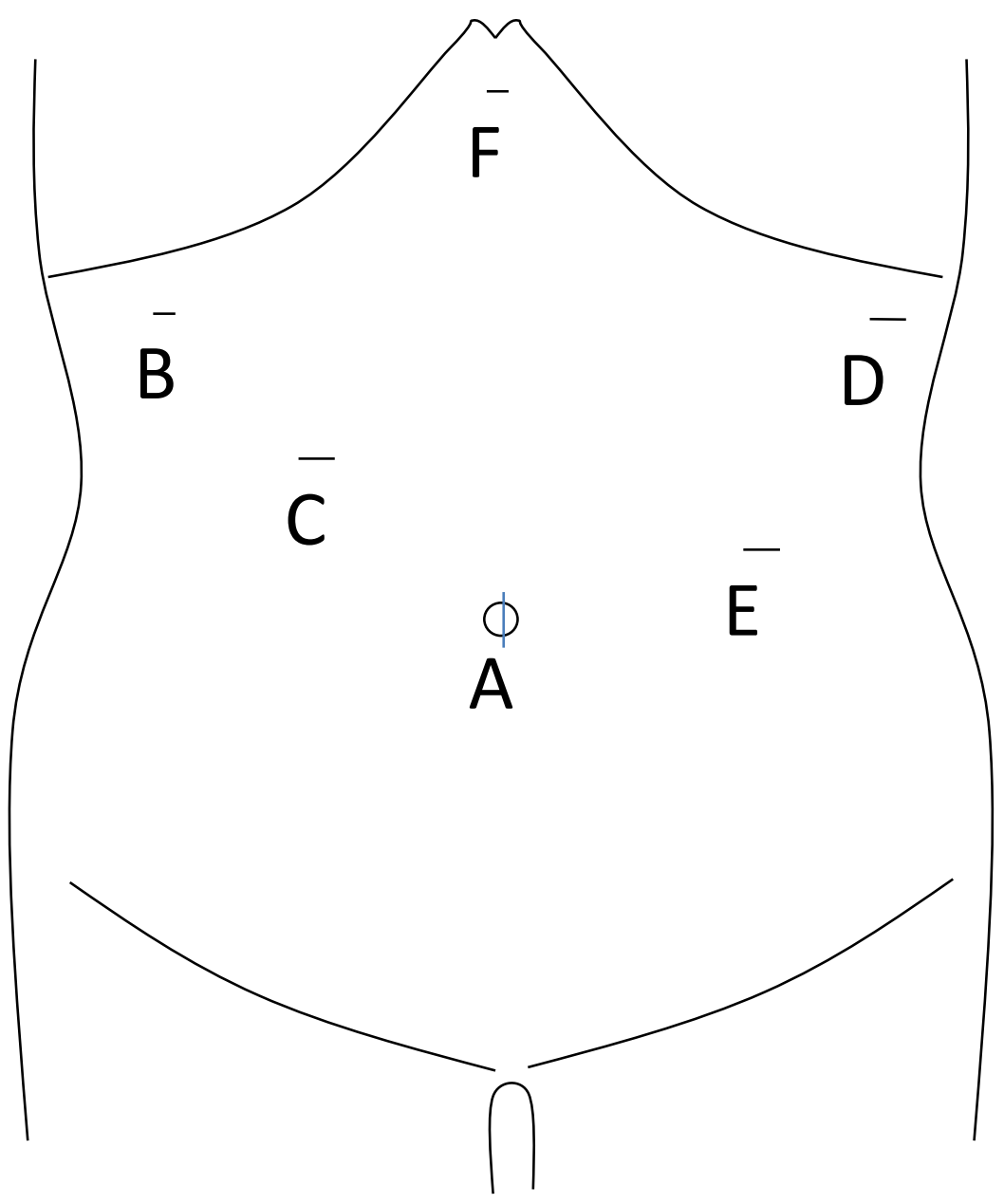


Figure 2

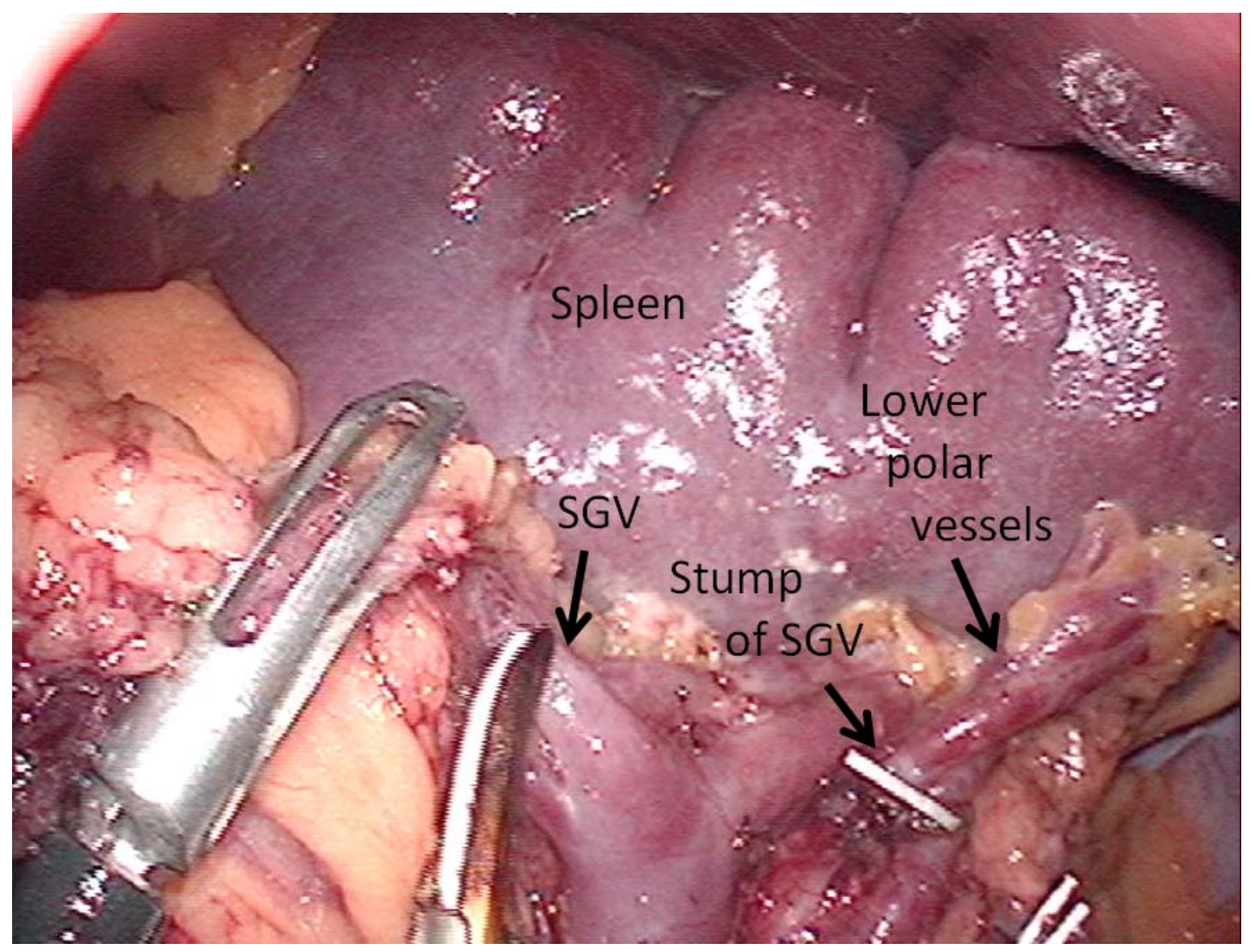




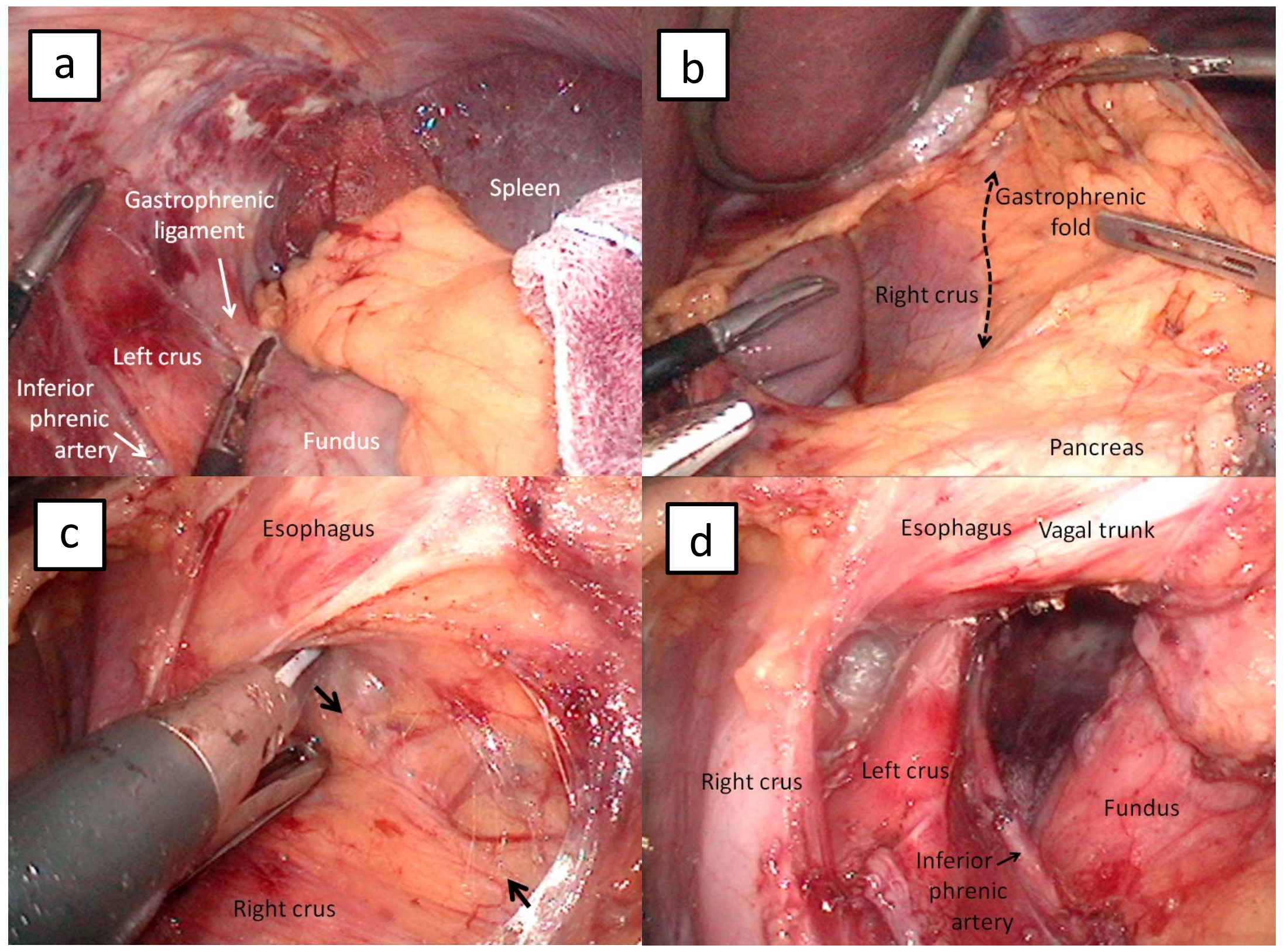




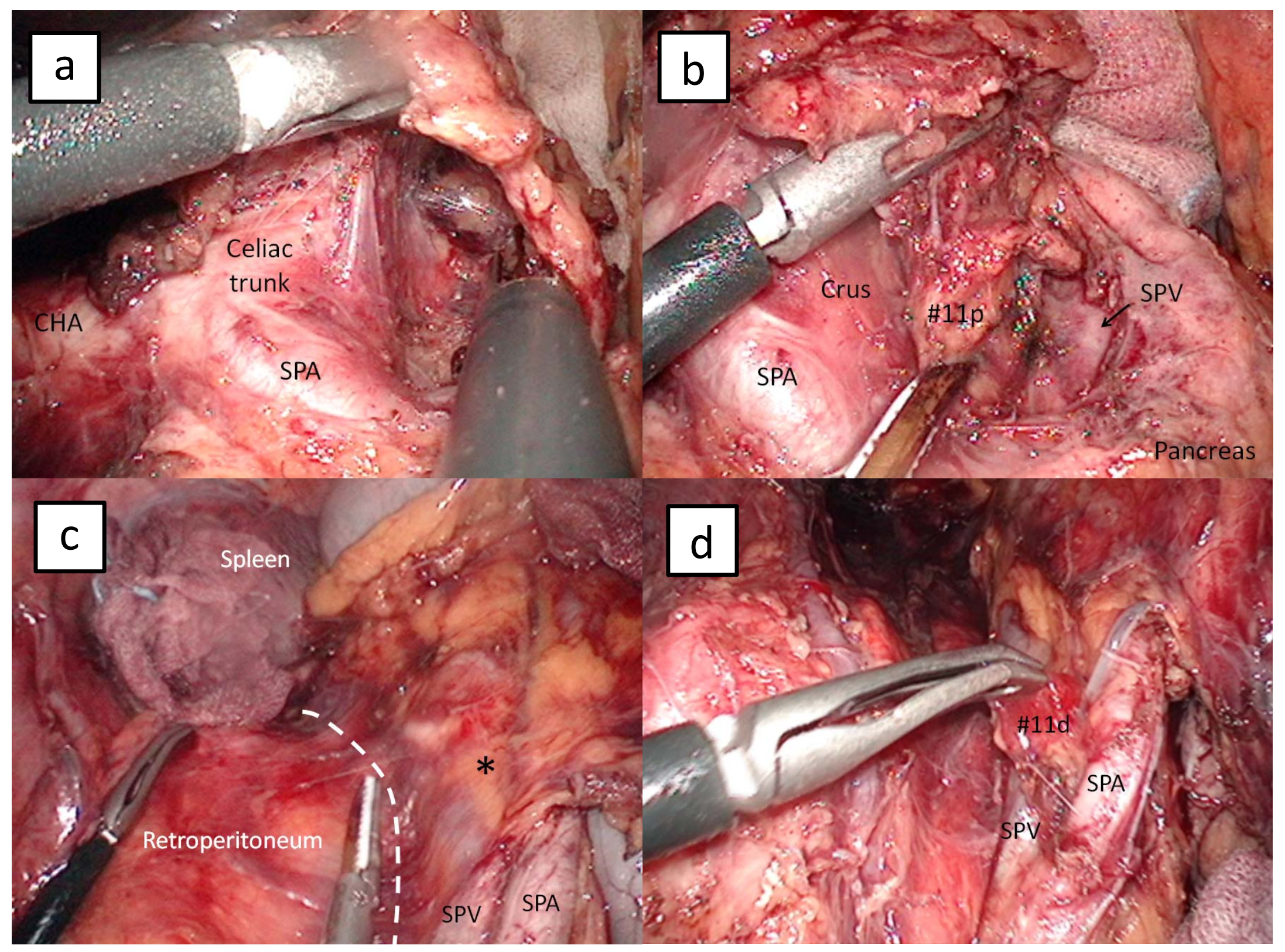




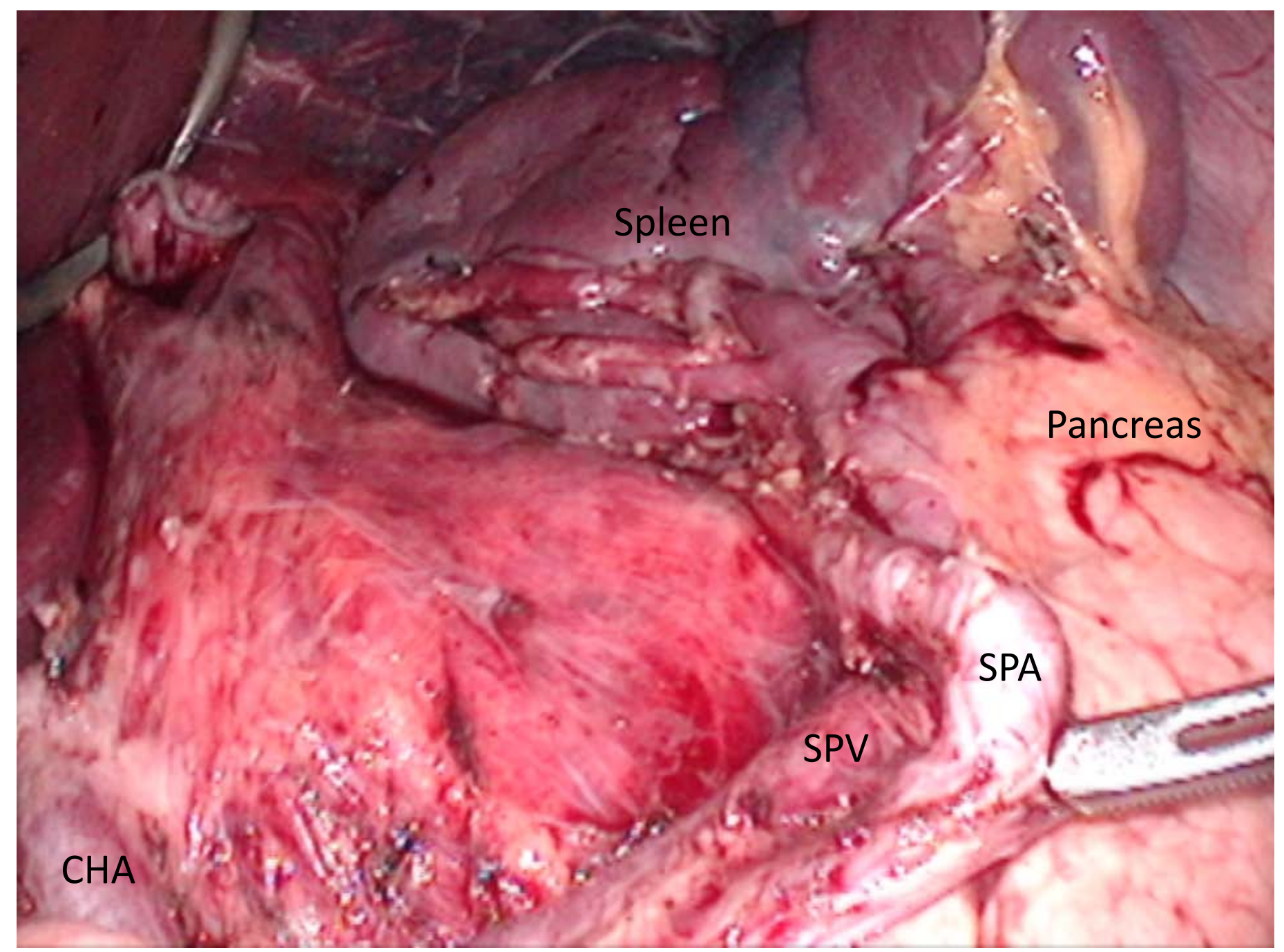

